\title{
Novel Fault Diagnosis Approach for Rolling-element Bearings Based on Bispectral Analysis
}

\author{
Ruige Zhang, Kun-Chieh Wang, ${ }^{*}$ Long Wu, and Hao Gao \\ School of Mechanical and Electric Engineering, Sanming University, Sanming, Fujian Province 365004, China
}

(Received September 6, 2021; accepted December 1, 2021)

Keywords: fault diagnosis, rolling-element bearing, variable operation conditions, bispectral analysis

Under variable operation conditions, the fault diagnosis of rolling-element bearings encounters the problems of ambiguous characteristic frequencies and inconsistent disturbance features. Conventional diagnosis methods have adopted extra hardware or signal preprocessing to overcome these problems, which usually resulted in difficulties in implementation and incorrect diagnosis. Here, we propose a novel approach based on the strategy of extracting features that are insensitive to changes in operation conditions. This may have the advantages of preventing interference from signal resampling and preprocessing. First, we derive the bispectral expressions of vibration signals for rolling-element bearings in use. Next, we use simulated and measured experimental data detected by numerous fluid and vibration sensors to identify the proposed model. Finally, experiments on the fault diagnosis of bearings are performed to validate our proposed approach. Results show that the proposed bispectral distribution method has the advantage of insensitivity to the operation conditions. In experiments involving three levels of fault severity, our proposed diagnosis model always correctly identified the fault type of rolling-element bearings under different operating conditions. With the advantages of directly extracting the insensitive bispectral features without the requirement of additional hardware and signal preprocessing, our proposed approach is simple and easy to implement, giving it good application prospects in engineering practice.

\section{Introduction}

Rolling-element bearings are a crucial component in rotating machinery but are also prone to fail in use. Therefore, their fault diagnosis plays a significant role in the study of reliability and safety for rotating machinery. ${ }^{(1)}$ Under fixed operation conditions, a traditional way to determine the bearing status is fault detection via the comparison of characteristic frequencies. However, when the operation conditions change, the performance of the fault detection method deteriorates owing to the feature inconsistency caused by rotation fluctuation. ${ }^{(2,3)}$ Such a fault detection method is obviously no longer suitable under variable operation conditions. In rotating machinery, variable operation conditions are common. For example, for wind turbines and mining equipment, the operating speed constantly changes during working. ${ }^{(4)}$ As indicated above, it is essential to find a suitable fault diagnosis method for bearings under variable operating conditions.

*Corresponding author: e-mail: m18316252102@126.com

https://doi.org/10.18494/SAM3636 
In the past, order tracking (OT) was a widely used method for bearing diagnosis in the case of a time-varying rotational speed. ${ }^{(5)}$ OT acquires phase information using extra hardware and by resampling vibration signals in the angle domain. Through signal resampling, non-stationary signals with equal time intervals can be transformed into stationary signals with equal angular intervals. Hence, the variable fault frequencies that occur at different rotational speeds can also be transformed into constant fault frequencies, thereby overcoming spectrum smearing. ${ }^{(6)}$ On the other hand, for rotating machinery, it is difficult and uneconomic to mount an auxiliary speed measurement device owing to the deterioration of the fluid working conditions and the complex mechanical structure. ${ }^{(7)}$ To overcome this problem, the methodology of tacholess order tracking (TOT) was proposed, which can be used to obtain a rotating machine's information from vibration signals while avoiding the difficulties in installing hardware. ${ }^{(8)}$

In the aforementioned OT and TOT methods, the accuracy of analysis results was significantly affected by speed variations and the use of interpolation techniques that were not easy to handle. ${ }^{(9,10)}$ To avoid these problems, some researchers proposed specific methods, without considering signal resampling, to directly deal with fluctuations in rotational speed. One of these proposed methods that adopted signal resampling was to apply generalized demodulation to map the instantaneous frequency trajectory to a straight line parallel to the time axis, from which a frequency spectrum was obtained without resampling. ${ }^{(11)}$ Another approach was to focus on methods of suppressing the speed fluctuation or rescaling the frequency axis because of the independence of the fault of a running bearing of its rotational speed. ${ }^{(12,13)}$

Recently, some intelligent methods for the fault diagnosis of roller bearings under variable operation conditions have been developed to overcome the problems of disturbance inconsistency for characteristic frequencies, such as the diagnosis methods of OT, transfer learning (TL), and feature selection (FS). ${ }^{(14-18)}$ In the OT diagnosis method, vibration signals are first resampled in the angle domain, and then the frequency-domain ${ }^{(14)}$ or time-domain ${ }^{(15)}$ features are extracted to train the diagnosis model. The TL diagnosis method utilizes techniques such as domain adaption, ${ }^{(16)}$ fine-tuning modeling, ${ }^{(17)}$ or deep learning ${ }^{(18)}$ to transform information between different operating conditions, thus improving the model adaptability to various operation conditions. The FS diagnosis method employs the disturbance attribute projection ${ }^{(19)}$ and singular value decomposition ${ }^{(20)}$ to select important feature vectors to improve the model recognition ability.

To investigate the effects of variable operation conditions on the fault diagnosis of bearings, an additional signal preprocessing procedure is usually adopted to overcome the problems of frequency smearing and disturbance inconsistency. This procedure makes fault diagnosis devices difficult to mount and introduces the problems of limited accuracy, ${ }^{(9)}$ improper parameters, ${ }^{(21)}$ and reduced reliability of data transfer. ${ }^{(22)}$ Therefore, in this study, to avoid extra data preprocessing, we propose a novel diagnosis method that can directly extract features that are insensitive to the variability of operation conditions. This should simplify the diagnosis method and allow a device to be easily mounted.

Generally, the fault diagnosis of running bearings adopts a technique that extracts features from the time, frequency, or time-frequency domain. The time-domain features include dimensional and dimensionless ones. Dimensional features include mean value, standard 
deviation, root mean square, and peak value, which are affected by the operation conditions. Dimensionless features include kurtosis, skewness, crest indicator, and clearance indicator. Many problems remain unsolved for time-domain features, such as how to optimally design an appropriate band-pass filter or select highly sensitive feature parameters. ${ }^{(23)}$ Time-frequencydomain features are normally viewed as non-stationary characteristic data and are not suitable for the diagnosis problems considered in this study because of their unsolved problems similar to those in the time domain. Therefore, in this study, we adopt frequency-domain features to analyze the status of running bearings, which can be used to filter Gaussian noise and understand a system's nonlinear behavior. ${ }^{(24)}$

In the research field of fault diagnosis involving vibrations, the bispectral method is commonly used to analyze amplitude as well as frequency-modulated signals. ${ }^{(25)}$ The bispectral method includes the frequency-shift bispectrum, ${ }^{(26)}$ cyclic bispectral slice, ${ }^{(27)}$ and bispectral distribution (BSD). ${ }^{(28)}$ Among them, the BSD has outstanding fault identification performance. Previous studies revealed that the BSD method can eliminate Gaussian noise and extract feature parameters more effectively by combining it with autoregression ${ }^{(29)}$ and singular value decomposition. ${ }^{(30)}$ For these reasons, we propose a novel fault diagnosis method based on the BSD to extract the vibration as well as system-invariant characteristics of rolling-element bearings under variable operation conditions. ${ }^{(31)}$

\section{Derivation of Bispectrum for Rolling-element Bearing}

\subsection{Signal model of rolling-element bearing}

A rolling-element bearing consists of rolling elements between inner and outer metal rings. As the bearing rotates, a defect on the surface of a certain roller element will periodically strike the surfaces of other roller elements that it comes in contact with. This undesired striking will produce an impulse that excites resonance in the whole bearing system. The resultant output of the vibration signal, $x(t)$, can be written as ${ }^{(32)}$

$$
x(t)=\sum_{i} A_{i} s\left(t-i T_{0}-\tau_{i}\right)+n(t)
$$

where $A_{i}$ is the amplitude of the modulating factor, $s(t)$ is the decaying oscillating component, $n(t)$ is the additive background noise from other vibration effects, $T_{0}$ is the repeated shock period, and $\tau_{i}$ is the minor random fluctuation time.

For simplification, it is assumed that the bearing vibration signal is expressed in the form of an oscillation wave related to the system decaying frequency. ${ }^{(32)}$ As a result, the decaying vibration signal $s(t)$ can be expressed as

$$
s(t)=e^{-\beta t} \cos \left(2 \pi f_{n} t\right),
$$


where $\beta$ and $f_{n}$ are the decay rate of the periodic shock and the resonance frequency of the bearing system, respectively.

The amplitude modulating factor $A_{i}$ can be written as

$$
A_{i}=A_{0}+A_{m} \cos \left(2 \pi f_{n} t+\psi\right) \text {, }
$$

where $\psi$ is the angle of the load zone, $f_{n}$ is the bearing rotation frequency, and $A_{0}$ and $A_{m}$ are the determining and alternating loads, respectively.

By examining the dynamic behavior resulting from the structure of the rolling-element bearing during working, it can be intuitively assumed that the known $\beta$ and $f_{n}$ are both related to the shock excitation locations. The fault diagnosis results can be expressed in terms of $s(t)$, and the amplitude of the modulating factor $A_{i}$ indicates the effect of the operation conditions.

\subsection{Bispectral expression of bearing vibration signal}

Let $x(t)$ be a one-dimensional signal whose bispectrum is defined as ${ }^{(33)}$

$$
B\left(f_{1}, f_{2}\right)=\lim _{w \rightarrow \infty} \frac{1}{w} \int_{-w / 2}^{w / 2} \int_{-\infty}^{\infty} \int_{-\infty}^{\infty} c_{3 x}\left(t, \tau_{1}, \tau_{2}\right) e^{-j 2 \pi f_{1} \tau_{1}} e^{-j 2 \pi f_{2} \tau_{2}} d \tau_{1} d \tau_{2} d t
$$

where $c_{3 x}\left(t, \tau_{1}, \tau_{2}\right)$ is the third-order cumulant of $x(t)$, expressed as

$$
\begin{aligned}
c_{3 x}\left(t, \tau_{1}, \tau_{2}\right)= & E\left[x(t) x\left(t+\tau_{1}\right) x\left(t+\tau_{2}\right)\right]-E[x(t)] E\left[x\left(t+\tau_{1}\right) x\left(t+\tau_{2}\right)\right] \\
& -E\left[x\left(t+\tau_{1}\right)\right] E\left[x\left(t+\tau_{2}\right) x(t)\right]-E\left[x\left(t+\tau_{2}\right)\right] E\left[x\left(t+\tau_{1}\right) x(t)\right] \\
& +2 E[x(t)] E\left[x\left(t+\tau_{1}\right)\right] E\left[x\left(t+\tau_{2}\right)\right] .
\end{aligned}
$$

Here, $E[\bullet]$ is the expectation operator.

For simplicity, we suppose that $A_{i}$ in Eq. (3) is a periodic and delta-correlated point function, $\tau_{i}$ in Eq. (4) is a zero-mean delta-correlated point parameter, $p_{\tau}(t)$ is a probability density function, and $\mathrm{n}(\mathrm{t})$ in Eq. (1) is a zero-mean stationary random function. We now substitute Eq. (1) into Eq. (5), then the third-order cumulant $c_{3 x}\left(t, \tau_{1}, \tau_{2}\right)$ can be obtained as

$$
\begin{aligned}
c_{3 x}\left(t, \tau_{1}, \tau_{2}\right)= & {\left[\sum_{i} \overline{A_{i}^{3}} s\left(t-i T_{0}\right) s\left(t-i T_{0}+\tau_{1}\right) s\left(t-i T_{0}+\tau_{2}\right)\right] * p_{\tau}\left(\tau_{i}\right) } \\
& -\sum_{i} \bar{A} \tilde{s}\left(t-i T_{0}\right)\left[\overline{A_{i}^{2}} s\left(t-i T_{0}+\tau_{1}\right) s\left(t-i T_{0}+\tau_{2}\right)\right] * p_{\tau}\left(\tau_{i}\right) \\
& -\sum_{i} \bar{A} \tilde{s}\left(t-i T_{0}+\tau_{1}\right)\left[\overline{A_{i}^{2}} s\left(t-i T_{0}\right) s\left(t-i T_{0}+\tau_{2}\right)\right] * p_{\tau}\left(\tau_{i}\right) \\
& -\sum_{i} \bar{A} \tilde{s}\left(t-i T_{0}+\tau_{2}\right)\left[\overline{A_{i}^{2}} s\left(t-i T_{0}\right) s\left(t-i T_{0}+\tau_{1}\right)\right] * p_{\tau}\left(\tau_{i}\right) \\
& +2 \sum_{i} \bar{A}_{i}^{3} s\left(t-i T_{0}\right) s\left(t-i T_{0}+\tau_{1}\right) s\left(t-i T_{0}+\tau_{2}\right),
\end{aligned}
$$


where $\overline{A_{i}}$ is the mathematical average of $A_{i}$ and $\tilde{s}(t)=s(t) * p_{\tau}(t)$ is the mathematical expectation of $s(t)$.

Substituting Eq. (6) into Eq. (4), we can acquire the formula of the bispectrum for vibration signal $x(t)$. The final form of $B$ is derived as

$$
\begin{aligned}
B\left(f_{1}, f_{2}\right)= & \sum_{i} \overline{A_{i}^{3}} S\left(f_{1}\right) S\left(f_{2}\right) S^{*}\left(f_{1}+f_{2}\right)-\sum_{i} \overline{A_{i}} \overline{A_{i}^{2}} S\left(f_{1}\right) S\left(f_{2}\right) \widetilde{S}^{*}\left(f_{1}+f_{2}\right) P_{\tau}\left(f_{1}+f_{2}\right) \\
& -\sum_{i} \overline{A_{i}} \overline{A_{i}^{2}} \widetilde{S}\left(f_{1}\right) S^{*}\left(f_{1}+f_{2}\right) S\left(f_{2}\right) P_{\tau}\left(f_{1}\right)-\sum_{i} \overline{A_{i}} \overline{A_{i}^{2}} \widetilde{S}\left(f_{2}\right) S^{*}\left(f_{1}+f_{2}\right) S\left(f_{1}\right) P_{\tau}\left(f_{2}\right) \\
& +\sum_{i} \bar{A}_{i}^{3} \widetilde{S}\left(f_{1}\right) \widetilde{S}\left(f_{2}\right) \widetilde{S}^{*}\left(f_{1}+f_{2}\right) \\
= & \overline{A^{3}} S\left(f_{1}\right) S\left(f_{2}\right) S^{*}\left(f_{1}+f_{2}\right)-\bar{A} \bar{A}^{2} S\left(f_{1}\right) S\left(f_{2}\right) \widetilde{S}^{*}\left(f_{1}+f_{2}\right) P_{\tau}\left(f_{1}+f_{2}\right) \\
& -\bar{A} \overline{A^{2}} \widetilde{S}\left(f_{1}\right) \widetilde{S}^{*}\left(f_{1}+f_{2}\right) S\left(f_{2}\right) P_{\tau}\left(f_{1}\right)-\bar{A} \overline{A^{2}} \widetilde{S}\left(f_{2}\right) \widetilde{S}^{*}\left(f_{1}+f_{2}\right) S\left(f_{1}\right) P_{\tau}\left(f_{2}\right) \\
& +2 \bar{A}^{3} \widetilde{S}\left(f_{1}\right) \tilde{S}\left(f_{2}\right) \widetilde{S}^{*}\left(f_{1}+f_{2}\right),
\end{aligned}
$$

where $\bar{A}$ is the summation of $\overline{A_{i}} \cdot S(f), \tilde{S}(f)$, and $P_{\tau}(f)$ are the Fourier transforms of $s(t), \tilde{s}(t)$, and $p_{\tau}(t)$, respectively.

Equation (7) shows that the BSD significantly depends on the properties of $S(f), \tilde{S}(f)$, and $P_{\tau}(f)$, and the amplitude of the bispectrum depends on $A_{i}$ and its mathematical expectation. From the above equations, it can be seen that the bispectra of vibration signals for bearings have the following features: The bispectra of different fault signals under different operating conditions are similar. In the above proposed signal models, the parameters $\beta$ and $f_{n}$ are identical, and the probability density functions of $p_{\tau}(t)$ are similar for the same type of fault signal. When the operation conditions change, the oscillating decay component $s(t)$, the mathematical average function $\tilde{s}(t)$, and the probability density function $p_{\tau}(t)$ do not apparently change with time. Similarly, the parameters $S(f), \tilde{S}(f)$, and $P_{\tau}(f)$ do not significantly change. In contrast, the amplitude parameters $\bar{A}, \overline{A^{2}}, \overline{A^{3}}$, and $\bar{A}^{3}$ significantly change with the operation conditions. As a result, under different operation conditions, the BSDs of fault signals are reasonably similar, but their bispectral amplitudes change.

The BSDs of the different fault signals are significantly different. In the aforementioned vibration signal model, the parameters $\beta$ and $f_{n}$ change with the fault type; thus, the oscillating decay component $s(t)$, the mathematical expectation $\tilde{s}(t)$, and the corresponding Fourier transforms $S(f)$ and $\widetilde{S}(f)$ also change, therefore resulting in different BSDs. 


\section{Fault Diagnosis of Running Rolling-element Bearings Under Different Operation Conditions}

\subsection{Bispectra of simulated vibration signals}

To examine our proposed model, we design a series of specific simulation experiments that include two types of fault signals and two operation conditions. The related experimental parameters are listed in Table 1. Moreover, a white Gaussian noise with a signal-to-noise ratio (SNR) of $-13 \mathrm{~dB}$ is added to represent the vibration disturbance from other components. The sampling frequency of the simulation signals is $500 \mathrm{~Hz}$.

In the bispectral analysis, we adopt a fragment of 1024 data points, which is divided into four sub-fragments. Each sub-fragment has 256 data points, and a total of 192 data points are set in every overlap region between two adjacent sub-fragments. To smooth the obtained data in the frequency domain, we use the Rao-Gabr window with a width of 5. The obtained bispectra of fault I under operation conditions I and II are respectively shown in Figs. 1(a) and 1(b), and the bispectrum of fault II under condition I is shown in Fig. 2.

Here, the obtained bispectra for various fault cases correspond to the resonance characteristics of the roller-element bearings in use during the same fault. For fault I signals, a dual-frequency distribution of $f_{1}=90 \mathrm{~Hz}$ and $f_{2}=90 \mathrm{~Hz}$ clearly appears under both conditions I and II. Similarly, Fig. 2 shows that a dual-frequency distribution of $f_{1}=120 \mathrm{~Hz}$ and $f_{2}=120 \mathrm{~Hz}$ also appears for

Table 1

Experimental parameters of simulated signals.

\begin{tabular}{llllll}
\hline \multirow{2}{*}{ Bearing status } & \multicolumn{5}{c}{ Model parameters } \\
\cline { 2 - 6 } & $A_{0}$ & $A_{m}$ & $f_{r}$ & $f_{n}$ & $\beta$ \\
\hline Condition I, fault I & 1 & 1 & 1.5 & 90 & 1 \\
Condition II, fault I & 0.8 & 1.1 & 2 & 90 & 1 \\
Condition I, fault II & 1 & 1 & 1.5 & 120 & 3 \\
\hline
\end{tabular}

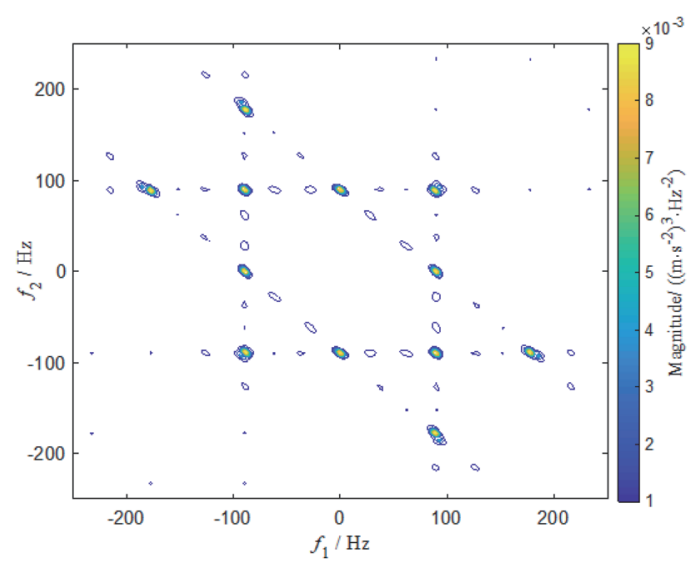

(a)

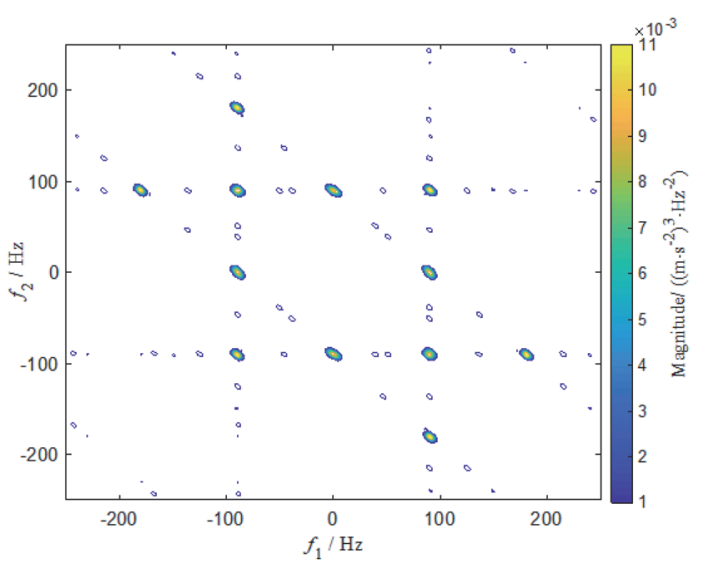

(b)

Fig. 1. (Color online) Bispectra of simulated signals for fault I under operation conditions (a) I and (b) II. 


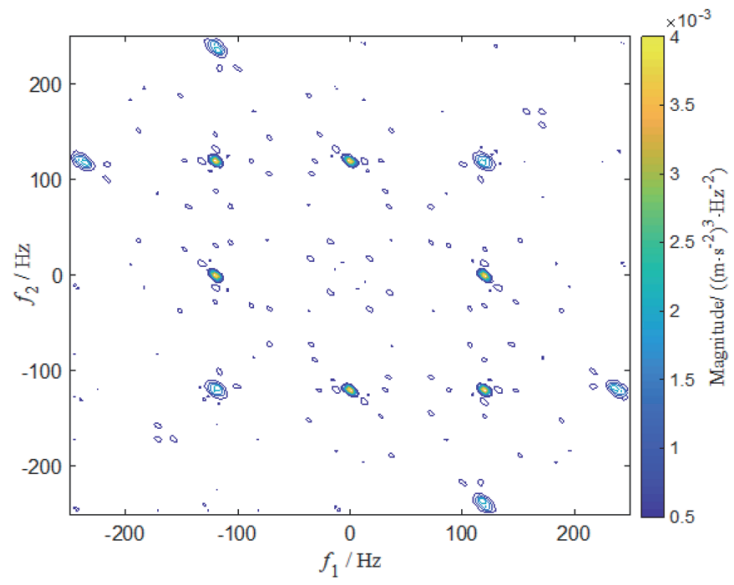

Fig. 2. (Color online) Bispectrum of simulated signals for fault II under operation condition I.

fault II signals under condition I. These dual-frequency distributions for signals of faults I and II coincide with their corresponding resonance frequencies.

Moreover, on one hand, it can be seen from Fig. 1 that the BSDs of operation conditions I and II are very similar. On the other hand, the BSD shown in Fig. 2 shows significant differences, not only in magnitude but also in pattern, from those in Figs. 1(a) and 1(b). This implies that, for the same fault signal, different operation conditions have little effect on the BSD. However, for different fault signals, the BSDs under different operation conditions have large differences.

\subsection{Bispectra of measured vibration signals}

The experimental data of vibration signals for running rolling-element bearings were adopted from Case Western Reserve University Bearing Data Center. The experimental setup is shown in Fig. 3(a). The experimental test stand consists of an electric motor, several fluid sensors (including torque transducers and dynamometers), and a control unit. A 6205-2RS test bearing [Fig. 3(b)] is mounted on the drive end. To simulate the fault signals of a roller-element bearing, some corrosion pits with different diameters on the bearing surface are made via electrodischarge machining and used to generate fault signals of vibration. An accelerometer is used as a vibration sensor, which is attached to the motor housing via a magnetic base. The vibration signals are collected via sensors at a sampling rate of 12000 per second via a 16-channel data recorder. The experiment involves four operation conditions, and the rotational speed and load of each condition are shown in Table 2.

Figure 4 shows the bispectra of a defective bearing with a 0.007 inch corrosion pit under the action of four different applied loads. Figure 4(a) shows the BSD of a normal roller-element bearing without any surface defect or applied load (operation condition I), which exhibits an apparent dual-frequency distribution at about $1 \mathrm{kHz}$. This implies that the rolling-element bearing does not induce any oscillation and the detected vibration mainly originates from the resonance of the whole machine body. Figure 4(b) shows the BSD of a defective roller-element bearing with a corrosion pit on the ball surface without any applied load (operation condition I), 


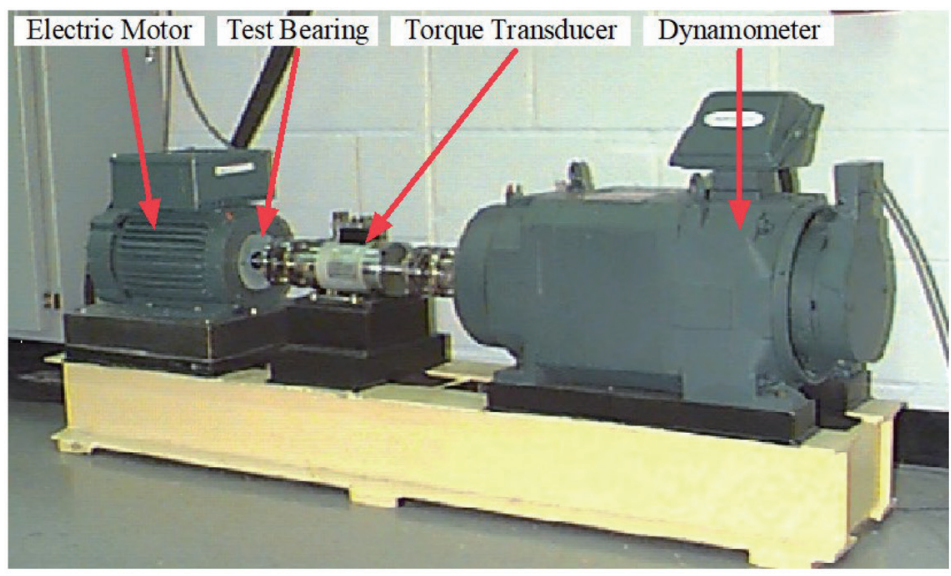

(a)

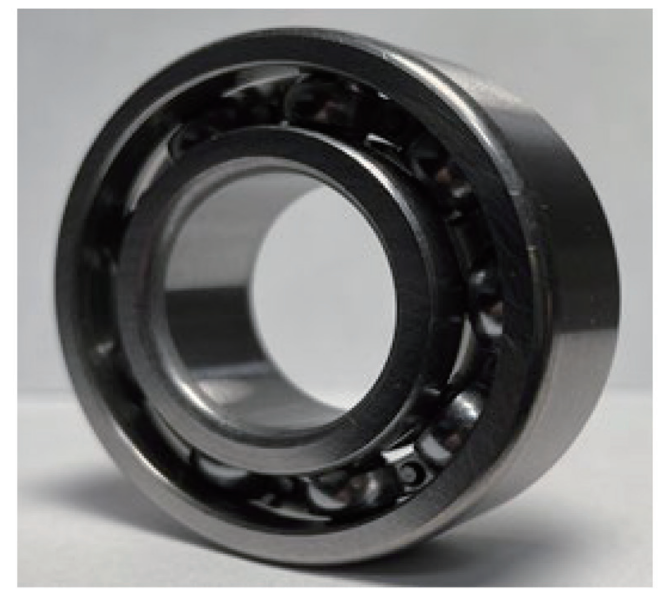

(b)

Fig. 3. (Color online) Experiment setup. (a) Overall test rig. (b) Test bearing. (33)

Table 2

Parameters of operation conditions.

\begin{tabular}{lcc}
\hline Operation condition & Load $(\mathrm{hp})$ & Rotational speed $(\mathrm{r} / \mathrm{min})$ \\
\hline I & $0(0 \mathrm{~W})$ & 1797 \\
II & $1(735 \mathrm{~W})$ & 1772 \\
III & $2(1470 \mathrm{~W})$ & 1750 \\
IV & $3(2205 \mathrm{~W})$ & 1730 \\
\hline
\end{tabular}

which is more complicated than that of a normal rolling-element bearing. This is because the defective rolling ball of the bearing scrapes its raceway while running, inducing resonance in the whole bearing system. When the defect is on the surface of the inner race, the effect of periodic scrapes seems to be larger than in the previous two cases. The resultant BSD is shown in Fig. 4(c), which is the most complex among the four operation conditions. When the defect is on the surface of the outer race, it is found from Fig. 4(d) that the peak value of the bispectrum is also 


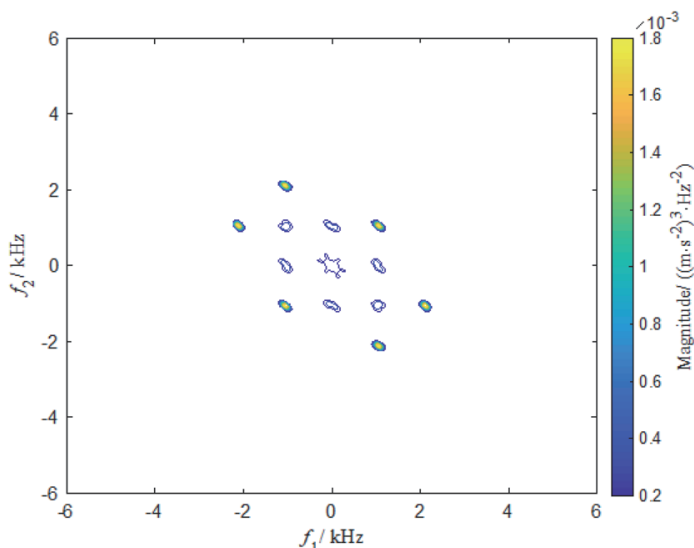

(a)

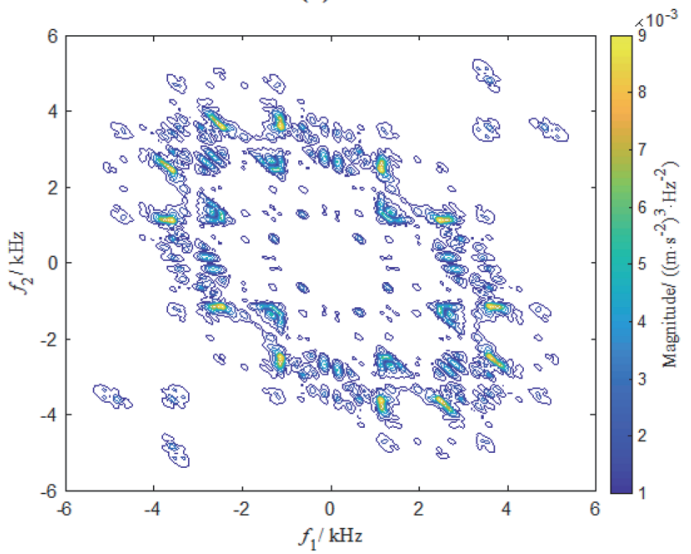

(c)

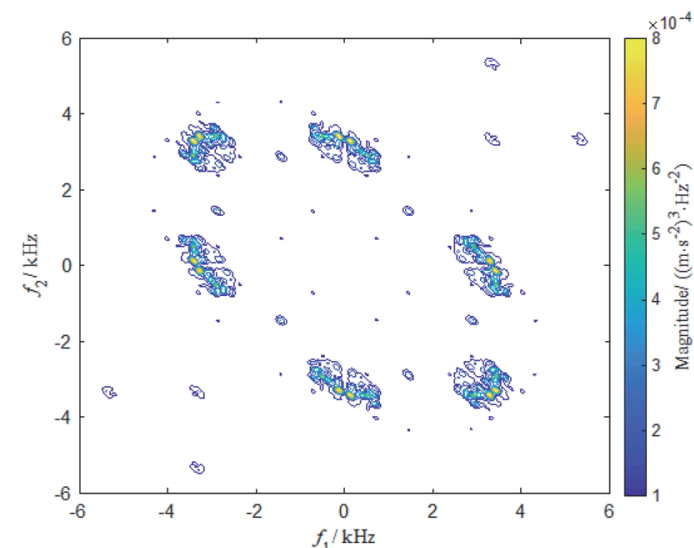

(b)

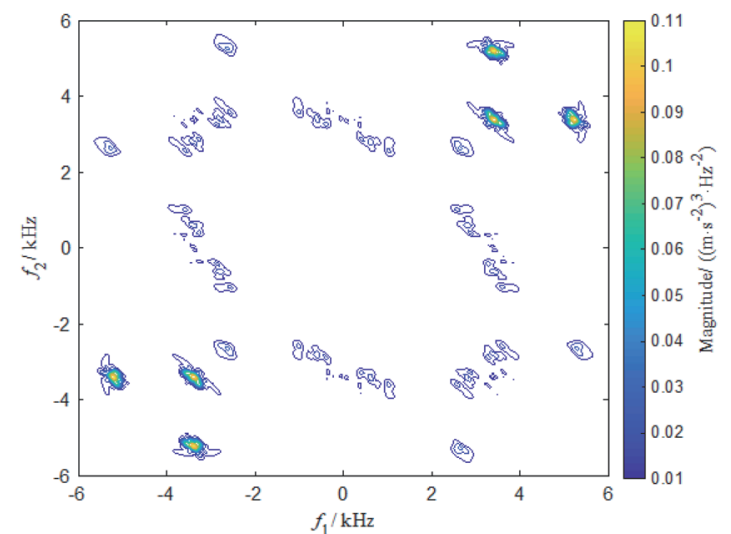

(d)

Fig. 4. (Color online) BSDs for four different defective rolling-element bearings under operation condition I. (a) Normal bearing without defect (normal state); (b) defective bearing with a 0.007 inch corrosion pit on the surface of a roller ball (ball fault); (c) defective bearing with a 0.007 inch corrosion pit on the surface of the inner race (inner race fault); (d) defective bearing with a 0.007 inch corrosion pit on the surface of the outer race (outer race fault).

the largest among all cases under consideration. This is because the defect is close to the sensor and may induce a larger mechanical oscillation.

Figure 5 shows the BSDs of the bearing with the inner race defect under operation conditions II, III, and IV. Although the overall distribution patterns are very similar, they are slightly different in details including the position as well as the magnitude. In other words, once the defect type is fixed, applying different loads may only induce local variations of the BSDs of bearings while their overall patterns remain almost the same.

In summary, for running rolling-element bearings, if their defect types are the same, then their BSDs of vibration are very similar for various operation conditions. In contrast, if their defect types are different, then their BSDs of vibration are markedly different, regardless of the operation condition. Therefore, we may conclude that BSDs can effectively reflect the vibration characteristics of running rolling-element bearings with or without defects. Thus, for the diagnosis of vibration problems of running rolling-element bearings, bispectral analysis exhibits the advantage of being insensitive to changes in operation conditions. This finding is a 


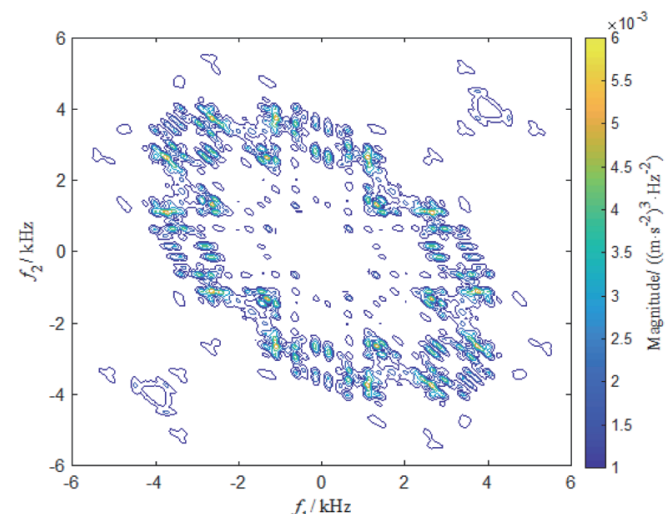

(a)

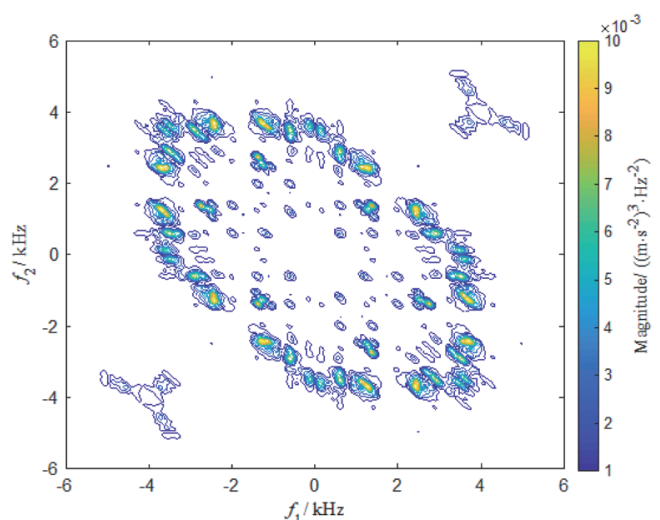

(b)

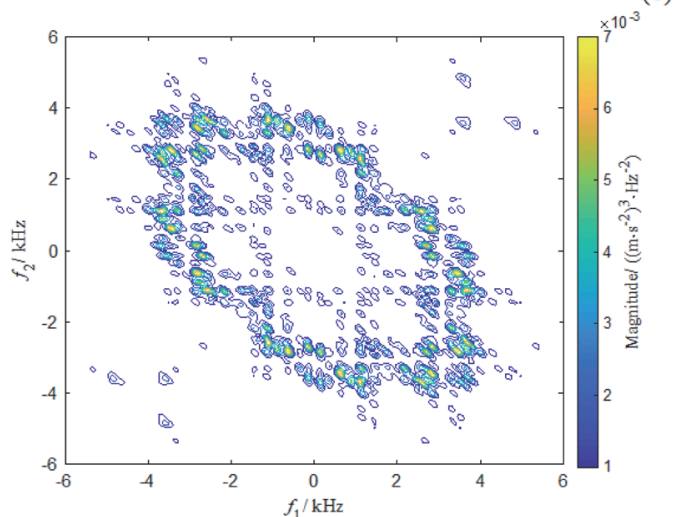

(c)

Fig. 5. (Color online) BSDs of inner race fault under operation conditions (a) II, (b) III, and (c) IV.

significant contribution toward applying bispectral analysis to the vibration diagnosis of problems of running rolling-element bearings under variable operation conditions.

\section{Fault Diagnosis of Running Rolling-element Bearings for Different Fault Severities}

To evaluate our proposed BSD analysis method in diagnosing the vibration of running rolling-element bearings, we design three groups of experiments based on the level of fault severity as follows. Group I: the defective bearing has a surface defect pit with a diameter of 0.007 inch, Group II: the defective bearing has a surface defect pit with a diameter of 0.014 inch, and Group III: the defective bearing has a surface defect pit with a diameter of $0.021 \mathrm{inch}$. The bispectra are calculated from experimental data via Eqs. (1)-(7) in Sect. 2. Moreover, to optimally handle the obtained experimental data, we use the decision tree method ${ }^{(18)}$ to classify the measured data and apply the fivefold cross-validation method ${ }^{(19)}$ to validate the diagnosis results.

The results of the three groups of experiments show that (1) for Group I, the diagnostic results obtained using our proposed BSD method have an identification accuracy of $100 \%$ for the four different operation conditions; (2) for Groups II and III, the accuracy of identification results and 
the standard deviations for the four operation conditions are satisfactory, as illustrated in Figs. 6 and 7, respectively. In both figures, the notation $L_{t} \rightarrow L_{i}$ indicates how the data are handled from training to testing, where $L_{t}$ and $L_{i}$ represent the operation condition numbers of the training and testing data, respectively.

The above diagnosis results with high identification accuracy indicate that our proposed BSD method can effectively identify the fault types of rolling-element bearings under different operation conditions. The diagnosis results also indicate that the identification accuracy of bearings for Group I ( 0.007 inch corrosion pit) is higher than those for Group II (0.014 inch corrosion pit) and Group III ( 0.021 inch corrosion pit). The reason is that the impact energy appearing in a running rolling-element bearing with a slight defect for Group I is smaller than

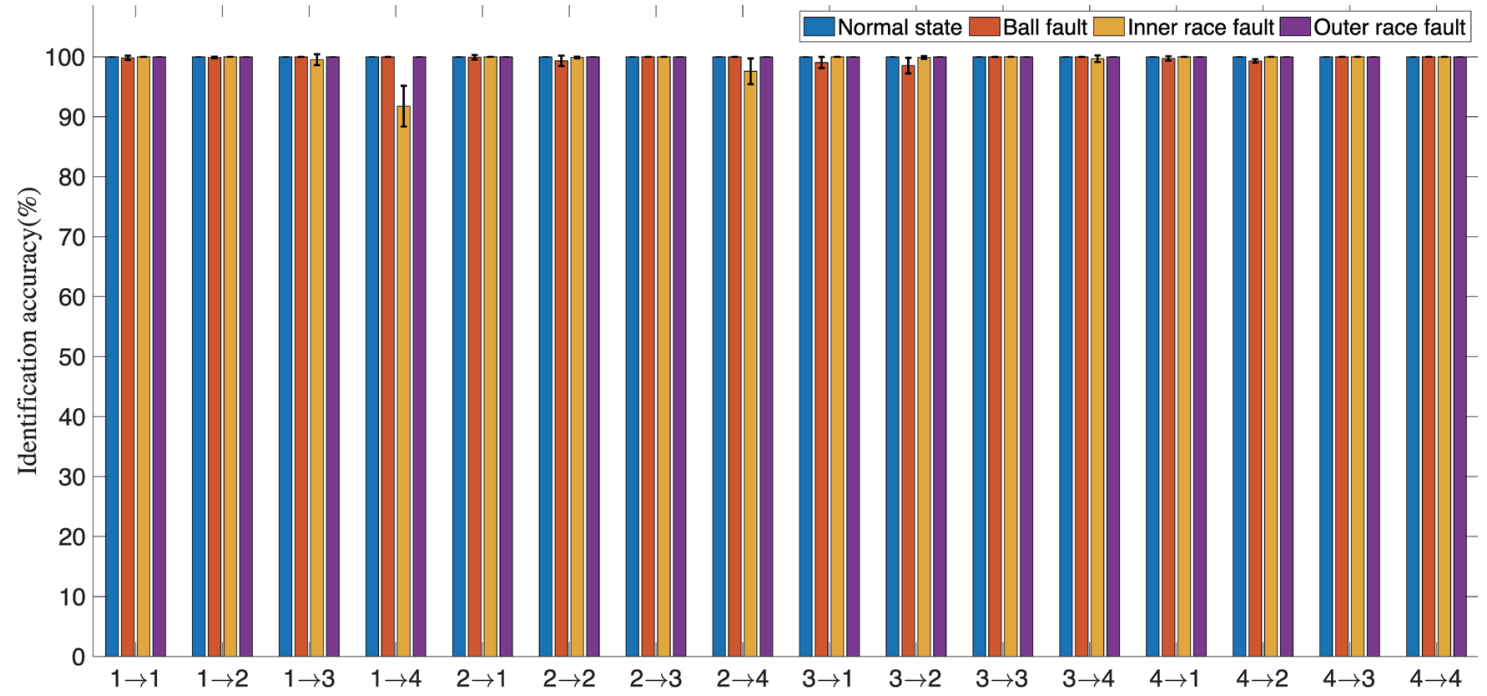

Fig. 6. (Color online) Identification accuracy of fault diagnosis for Group II under different operation conditions.

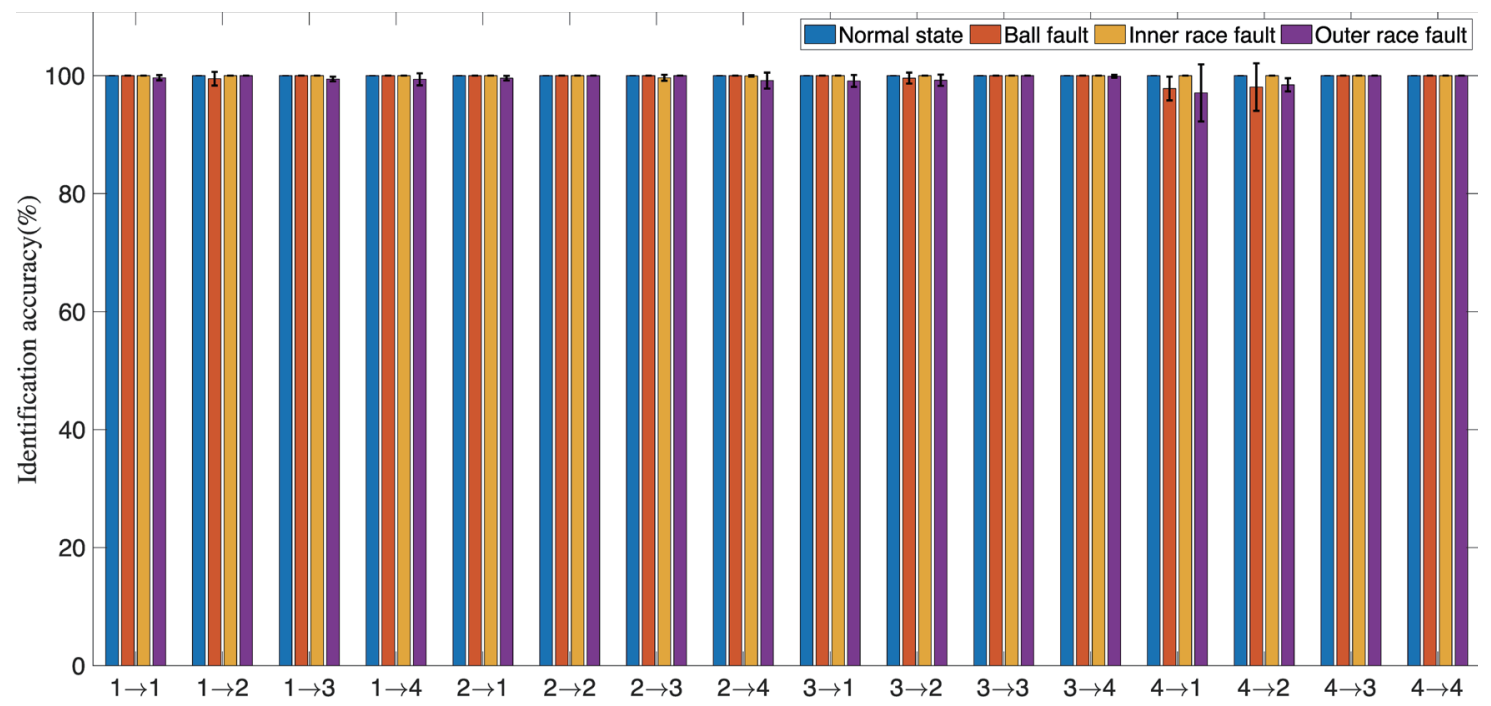

Fig. 7. (Color online) Identification accuracy of fault diagnosis for Group III under different operation conditions. 


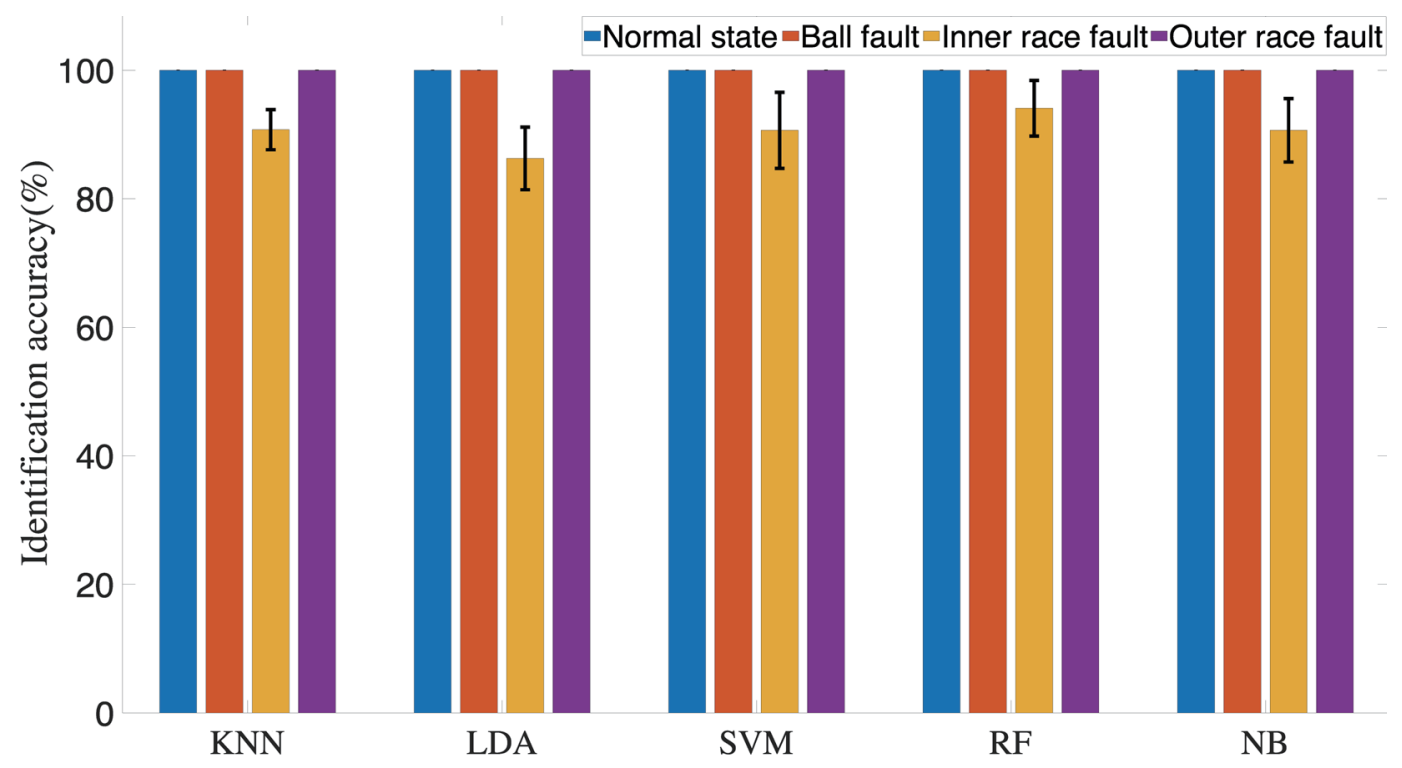

Fig. 8. (Color online) Identification accuracy of fault diagnosis using KNN, LDA, SVM, RF, and NB methods for Group III under different operation conditions.

those for the other two groups (Groups II and III), whose bearings have larger defects, and thus the disturbance occurring in the signals with high impact energy reduces the diagnosis accuracy. On the basis of the above discussion, it is confirmed that, for running rolling-element bearings, our proposed BSD analysis method can extract optimal and simple vibration features from signals and therefore has good recognition accuracy.

To further demonstrate the superiority of our proposed BSD analysis, we introduce five other commonly used methods for signal identification: the K-nearest neighbor (KNN) method, ${ }^{(30)}$ linear discriminant analysis (LDA) method, ${ }^{(30)}$ support vector machine (SVM) method, ${ }^{(34)}$ random forest (RF) method, ${ }^{(35)}$ and naive Bayes (NB) method. ${ }^{(36)}$ Figure 8 shows the results of diagnosis using the above five identification methods for Group II ( 0.014 inch corrosion pit), $1 \rightarrow 4$. It can be seen that these five identification schemes all show good overall reliability and high recognition ability with an identification accuracy of nearly $100 \%$ for the normal state, ball fault, and outer race fault conditions. However, for the fault diagnosis of the inner race state, the identification accuracies of the KNN, LDA, SVM, RF, and NB methods are 90.7, 86.3, 90.6, 94.1 , and $90.6 \%$, respectively, whereas our proposed BS method has an identification accuracy of $100 \%$, about 1.11 times as accurate as the average of the other five schemes $(90.46 \%)$.

\section{Conclusion}

Regarding the fault diagnosis problem of running rolling-element bearings, previous frequently used methods cannot accurately recognize faults owing to the ambiguity originating from data sampling and processing procedures as well as the difficulty in dealing with timedomain signals of vibration. However, our proposed BSD analysis method can effectively extract the inherent and invariant characteristics of running rolling-element bearings while avoiding the 
interference occurring in the above situations. Through testing via a series of experiments, it has been demonstrated that the BSD of rolling-element bearings in use is negligibly affected by the variations in operation conditions, making it particularly suitable as a diagnosis method for determining the fault type of rolling-element bearings in use. Comparisons with the commonly used KNN, LDA, SVM, RF, and NB methods show that our proposed BSD method has the highest recognition ability. Furthermore, our proposed BSD method does not require any extra hardware or signal preprocessing procedure, and is simple and easy to implement. Overall, the proposed BSD method has excellent application potential in practical engineering diagnosis problems involving rolling-element bearings.

\section{Acknowledgments}

This work was supported by the Key Project of the Natural Science Foundation of the Education Department of Fujian Province (JZ160476), the Major Project of the Sanming Science and Technology Plan (2019-G-7), the Project of the National Key R\&D Plan of the Ministry of Science and Technology (2020YFB2010103), the Project of the Educational Office of Fujian Province (IRTSTFJ), the Program for the Innovative Research Team in Science and Technology in Fujian Province University, Key Laboratory of Intelligent Equipment of Fujian Higher Education Institute, and the Project of Sanming University of Fujian Province (19YG05).

\section{References}

1 R. Liu, B. Yang, E. Zio, and X. Chen: Mech. Syst. Sig. Process. 108 (2018) 33. https://doi.org/10.1016/j. ymssp.2018.02.016

2 A. B. Ming, W. Zhang, Z. Y. Qin, and F. L. Chu: Mech. Syst. Sig. Process. 76-77 (2016) 367. https://doi. org/10.1016/j.ymssp.2016.02.021

3 S. J. Pan and Q. Yang: IEEE Trans. Knowledge Data Eng. 22 (2010) 1345. https://doi.org/10.1109/ TKDE.2009.191

4 Y. Hu, X. Tu, F. Li, H. Li, and G. Meng: J. Sound Vib. 409 (2017) 241. https://doi.org/10.1016/j.jsv.2017.08.003

5 P. Borghesani, R. Ricci, S. Chatterton, and P. Pennacchi: Mech. Syst. Sig. Process. 38 (2013) 23. https://doi. org/10.1016/j.ymssp.2012.09.014.

6 C. Mishra, A. K., Samantaray, and G. Chakraborty: Mech. Syst. Sig. Process. 72-73 (2016) 206. https://doi. org/10.1016/j.ymssp.2015.10.019

7 Y. Wang, G. Xu, A. Luo, L. Liang, and K. Jiang: J. Sound Vib. 367 (2016) 233. https://doi.org/10.1016/j. jsv.2015.12.041

8 S. Schmidt, P. S. Heyns, and J. P. de Villiers: Mech. Syst. Sig. Process. 100 (2018) 630. https://doi.org/10.1016/j. ymssp.2017.07.053

9 K. R. Fyfe and E. D. S. Munck: Mech. Syst. Sig. Process. 11 (1997) 187. https://doi.org/10.1006/mssp.1996.0056

10 W. Cheng, R. X. Gao, J. Wang, T. Wang, W. Wen, and J. Li: Mech. Syst. Sig. Process. 48 (2014) 92. https://doi. org/10.1016/j.ymssp.2014.03.004

11 D. Zhao, J. Li, W. Cheng, and W. Wen: J. Sound Vib. 378 (2016) 109. https://doi.org/10.1016/j.jsv.2016.05.022

12 Y. Wang, G. Xu, Q. Zhang, D. Liu, and K. Jiang: J. Sound Vib. 348 (2015) 381. https://doi.org/10.1016/j. jsv.2015.03.018

13 Y. Wang, P. W. Tse, B. Tang, Y. Qin, L. Deng, T. Huang, and G. Xu: Mech. Syst. Sig. Process. 122 (2019) 580. https://doi.org/10.1016/j.ymssp.2018.12.037

14 Y. Yang Yu, H. Wang, J. Cheng, and K. Zhang: Measurement 46 (2013) 2306. https://doi.org/10.1016/j. measurement.2013.03.023

15 X. Zhou, X. Zhang, W. Zhang, and X. Xia: Measurement 138 (2019)162. https://doi.org/10.1016/j. measurement.2019.01.063

16 Tong Zhe, Wei Li, Bo Zhang, and Meng Zhang: Shock Vib. 1 (2018) 1. https://doi.org/10.1155/2018/6714520 
17 H. Te, C. Liu, W. Yang, and D. Jiang: ISA Trans. 97 (2020) 269. https://doi.org/10.1016/j.isatra.2019.08.012

18 W. Qian, S. Li, and X. Jiang: Pattern Recognit. 96 (2019) 106993. https://doi.org/10.1016/j.patcog.2019.106993

19 W. Huang, J. Cheng, and Y. Yang: Mech. Syst. Sig. Process. 114 (2019) 165. https://doi.org/10.1016/j. ymssp.2018.05.015.

20 T. Ye, J. Ma, C. Lu, and Z. Wang: Mech. Mach. Theor. 90 (2015) 175. https://doi.org/10.1016/j. mechmachtheory.2015.03.014

21 H. Huan, N. Baddour, and M. Liang: J. Sound Vib. 414 (2018) 43. https://doi.org/10.1016/j.jsv.2017.11.005

22 Y. Chang, Y. Xu, H. Yu, Y. Yan, and Y. Liu: Knowledge-Based Syst. 203 (2020) 1. https://doi.org/10.1016/j. knosys.2020.106132

23 Y. Lei, B. Yang, X. Jiang, F. Jia, N. Li, and A. K. Nandi: Mech. Syst. Sig. Process. 138 (2020) 106587. https:// doi.org/10.1016/j.ymssp.2019.106587

24 Z. K. Peng, W. M. Zhang, B. T. Yang, G. Meng, and F. L. Chu: Mech. Syst. Sig. Process. 36 (2013) 456. https:// doi.org/10.1016/j.ymssp.2012.10.010.

25 G. Shen, S. McLaughlin, X. Y. Cheng, and P. White: Mech. Syst. Sig. Process. 43 (2014) 76. https://doi. org/10.1016/j.ymssp.2013.08.023.

26 G. Dong, J. Chen, and F. Zhao: J. Sound Vib. 339 (2015) 396. https://doi.org/10.1016/j.jsv.2014.11.015

27 Y. J. Zhou. G. Chen, M. Dong, W. B. Xiao, and Z. Y. Wang: Mech. Syst. Sig. Process. 26 (2012) 229. https://doi. org/10.1016/j.ymssp.2011.07.006

28 L. Jiang, Y. Liu, X. Li, and S. Tang: Meas.: J. Int. Meas. Confederation 44 (2011) 1284. https://doi.org/10.1016/j. measurement.2011.03.024

29 J. Guo, Z. Hao, D. Zhen, Z. Shi, F. Gu, and D. B. Andrew: Meas.: J. Int. Meas. Confederation 151 (2020) 107240. https://doi.org/10.1016/j.measurement.2019.107240

30 S. Luo, J. Cheng, M. Zeng, and Y. Yang: Meas.: J. Int. Meas. Confederation 87 (2016) 38. https://doi. org/10.1016/j.measurement.2016.01.006

31 C. K. Christopher and A. H. Matthew: Signal Process. 165 (2019) 357. https://doi.org/10.1016/j. sigpro.2019.07.007

32 F. Cong, J. Chen, G. Dong, and M. Pecht: J. Sound Vib. 332 (2013) 2081. https://doi.org/10.1016/j.jsv.2012.11.029

33 Seeded Fault Test Data: Case Western Reserve University Bearing Data Center, https://csegroups.case.edu/ bearingdatacenter/home (accessed 15 August 2020).

34 M. Hernández, E. Fidel, and C. M. Oscar: Mech. Syst. Sig. Process. 22 (2008) 588. https://doi.org/10.1016/j. ymssp.2007.09.003

35 D. Chen, V. Montano, L. Huo, and G. Song: Meas.: J. Int. Meas. Confederation 163 (2020) 107869. https://doi. org/10.1016/j.measurement.2020.107869

36 Y. Pu, D. B. Apel, and B. J. Lingga: Sustain. Mining 17 (2018) 158. https://doi.org/10.1016/j.jsm.2018.07.004 